\title{
Neutrophils-Lymphocytes Ratio (NLR) and Platelet-Lymphocytes Ratio (PLR) as Prognostic Factor of Osteosarcoma Patients at H. Adam Malik Hospital 2013 - 2018
}

\author{
Stefan Andhika ${ }^{\mathrm{a}}$, Pranajaya Dharma Kadar ${ }^{\mathrm{b}}$, Muhammad Hidayat Siregar \\ ${ }^{a}$ Faculty of Medicine Universitas Sumatera Utara, Jalan Dr. T. Mansur No. 9, Medan 20155, Indonesia \\ ${ }^{\mathrm{b}}$ Faculty of Medicine Universitas Sumatera Utara, Jalan Dr. T. Mansur No. 9, Medan 20155, Indonesia \\ ${ }^{c}$ Faculty of Medicine Universitas Sumatera Utara, Jalan Dr. T. Mansur No. 9, Medan 20155, Indonesia
}

\begin{abstract}
:
Introduction : Several systemic markers are used to predict the prognosis of several malignant tumours, but no study has been done to observe the correlation between inflammatory markers and 2-year survival rate of osteosarcoma patients. The purpose of this study is to identify the correlation between NLR and PLR with osteosarcoma prognosis and the patient's 2-year survival rate.

Methodology : This is a retrospective study with a diagnostic research design. Data of patients who were diagnosed with osteosarcoma from January 2013 - December 2018 in Haji Adam Malik Hospital is collected from their medical record or through by-phone interview to assess the patient's management history and progression post-treatment.

Result : A total of 40 samples were included in this study. Using the Kaplan-Meier graph, 2-year survival rate was analysed according to age, gender, tumour location, metastatic status, previous treatment, NLR, and PLR value. Only 1 subject has a survival rate of more than 2 years. In relation to NLR and PLR, it is shown that subjects with lower values have higher survival rate as compared to subjects with higher values.

Discussion : ROC analysis was performed to see the incidence of osteosarcoma by observing AUC determine its significance. The AUC NLR value for osteosarcoma $(0.972$, p-value $0.002,95 \%$ CI, 0.919-1000) was obtained, and the NLR cut-off value was 1.41 (sensitivity 75\%; specificity 97\%). The AUC PLR value for osteosarcoma (0.944, p-value $0.004,95 \%$ CI $0.871-1,000)$ and cutoff value of 97.477 (sensitivity $75 \%$, specificity $89 \%$ ) were obtained. The 2 -year survival rate of osteosarcoma patients was higher, namely 29 months in subjects with low NLR values, 13 months in subjects with high NLR, 17 months in subjects with low PLR values, and 14 months in subjects with high PLR values.
\end{abstract}

Keywords: Osteosarcoma; malignant tumour, prognostic marker

\section{Introduction}

Osteosarcoma is a malignant bone tumour derived from primitive mesenchymal cells and consists of malignant cells that produce bone and the osteoid matrix. It is the most common primary malignant bone tumour and occurs most frequently in the metaphysis of long bones, such as the distal femur, proximal tibia, and proximal humerus during the second decade of life. (Ando et al, 2013, p.591-616; Salter, 1999; Raymond et al, 2002)

The incidence of osteosarcoma in the entire population is about 4-5 per 1,000,000 population. (Raymond, 2002) This is higher in adolescents (adolescents) as much as 8-11 per 1,000,000 population per year at the age of 15-19 years. (Messerschmitt et al, 2009, p.515-527, Ritter et al, 2010, p.320-325)

Conservatively, osteosarcoma is treated by amputation surgery due to its aggressive and progressive nature. (Cortes et al, 2005, p.5-8; Mankin et al, 2004, p.286-291) With the development of chemotherapy which significantly reduces the possibility of metastasis as well as improving prognosis, limb salvage 
procedures have a success rate equal / higher compared to amputation surgeries. (Bacci et al, 2002, p.88-92; Ebeid et al, 2005, p.57-61, Lewis, 2007, p.57-61) Recently there are also emerging evidence suggesting that systemic inflammatory response may be a prognostic biomarker in several types of tumour. Some inflammatory markers such as thrombocytosis, leucocytosis, neutrophil to lymphocyte ratio (NLR), or platelet to lymphocyte ratio (PLR) values can be prognostic factors for several types of tumour, however, the evidence suggesting the effect of neutrophils to lymphocytes ratio on the prognosis of osteosarcoma patients has not been reported.(Xia et al, 2016, p.127)

\section{Experimental Methodology}

This research is a retrospective study with a diagnostic research design to define the correlation between neutrophile, lymphocyte, and platelet count and the subject's prognosis in a 2-year setting. This research was conducted at The Faculty of Medicine, University of North Sumatera / Haji Adam Malik Hospital, North Sumatera, Indonesia from October 2020 to November 2020. The sample chosen for this study uses a total sampling method, including all patients diagnosed with osteosarcoma who either received or did not receive conservative or surgical therapy, admitted from January 2013 up until December 2018 who met the inclusion and exclusion criteria. The inclusion criteria for this study were all patients who either received adjuvant chemotherapy post-operation or did not receive adjuvant chemotherapy post-operation. The exclusion criteria for this study are all samples who are lost to follow-up.

Data of the samples chosen for this study was collected from their medical records and patient interview. Data collected from their medical records include the patient's identity, previous diagnosis, osteosarcoma grading, and previous treatment. Patient interview is done by phone to ask the patient's current condition and history after treatment. Data obtained was then compiled, mean and standard deviation was measured, and evaluated using SPSS version 25.0 using Kaplan-Meier test to measure the sample's survival rate.

\section{Results}

A total of 40 medical records were assessed for eligibility and met the inclusion criteria. Respondents were generally male (75\%). All descriptive parameter data are presented in table 1. 
Table 1. Basic Characteristics of Respondents

\begin{tabular}{|c|c|c|c|}
\hline & Variable & $\mathbf{N}(\%)$ & Med (Min-Max) \\
\hline Age & & & $18(7-73)$ \\
\hline \multicolumn{4}{|l|}{ Sex } \\
\hline & Male & $30(75 \%)$ & \\
\hline & Female & $10(25 \%)$ & \\
\hline \multicolumn{4}{|c|}{ Tumor location } \\
\hline & Proximal humerus & $3(7.5 \%)$ & \\
\hline & Proximal femur & $8(20 \%)$ & \\
\hline & Distal femur & $12(30 \%)$ & \\
\hline & Proximal tibia & $14(35 \%)$ & \\
\hline & Proximal fibula & $3(7.5 \%)$ & \\
\hline \multicolumn{4}{|l|}{ Metastatic } \\
\hline & Yes & $24(60 \%)$ & \\
\hline & No & $16(40 \%)$ & \\
\hline \multicolumn{4}{|l|}{ Treatment } \\
\hline & Palliative care & $1(2.5 \%)$ & \\
\hline & Core biopsy & $1(2.5 \%)$ & \\
\hline & Open biopsy & $2(5 \%)$ & \\
\hline & Fine needle aspiration biopsy & $1(2.5 \%)$ & \\
\hline & Above knee amputation (AKA) & $24(60 \%)$ & \\
\hline & Four-quarter amputation & $1(2.5 \%)$ & \\
\hline & Limb salvage procedure & $2(5 \%)$ & \\
\hline & Hip disarticulation & $8(20 \%)$ & \\
\hline NLR & & & $3.90(0.71-45.01)$ \\
\hline PLR & & & $165.287(6.270-807.575)$ \\
\hline Survival rate & & & $14.5(9-34)$ \\
\hline
\end{tabular}

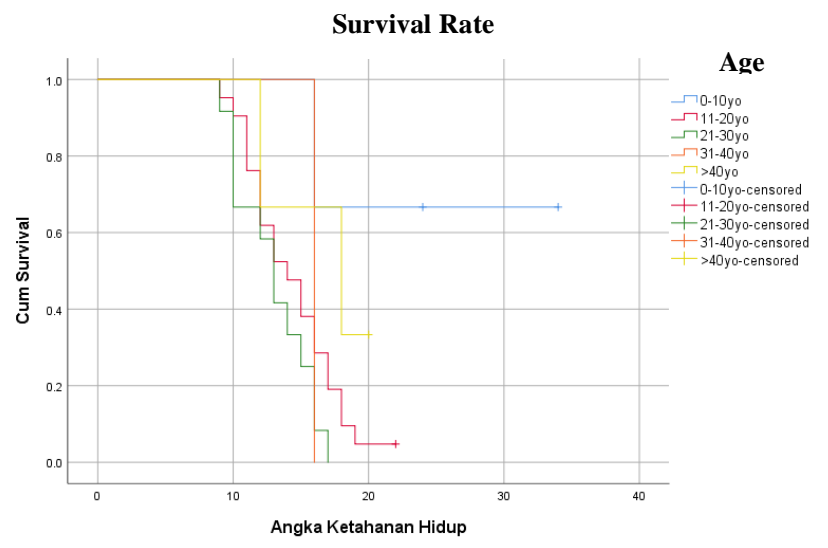

Figure 1. Kaplan-Meier Graph Showing 2-years Survival Rate by Age Group 
The 2-years survival rate was analyzed with a Kaplan-Meier graph. In this study, we look for 2-years survival rate by age, gender, location of the tumor, metastasis / no metastasis, treatment, NLR, and PLR value. From 40 respondents, only 1 subject has a survival rate of more than 2 years, in the $0-10$ years old age group (mean survival rate \pm 28 months). It is greater than any other age group. The same goes to the mean survival, which is highest on the $0-10$ years old age group (Figure 1). Mean survival rate by gender, female ( \pm 18 months) patients show the 2-years survival rate is better than male ( \pm 14 months) (Figure 2$)$. The 2-year survival rate of osteosarcoma located on proximal humerus ( \pm 20 months) is better than other tumour locations; proximal femur $( \pm 14$ months), distal femur, proximal tibia, and proximal fibula $( \pm 15$ months) (Figure 3).

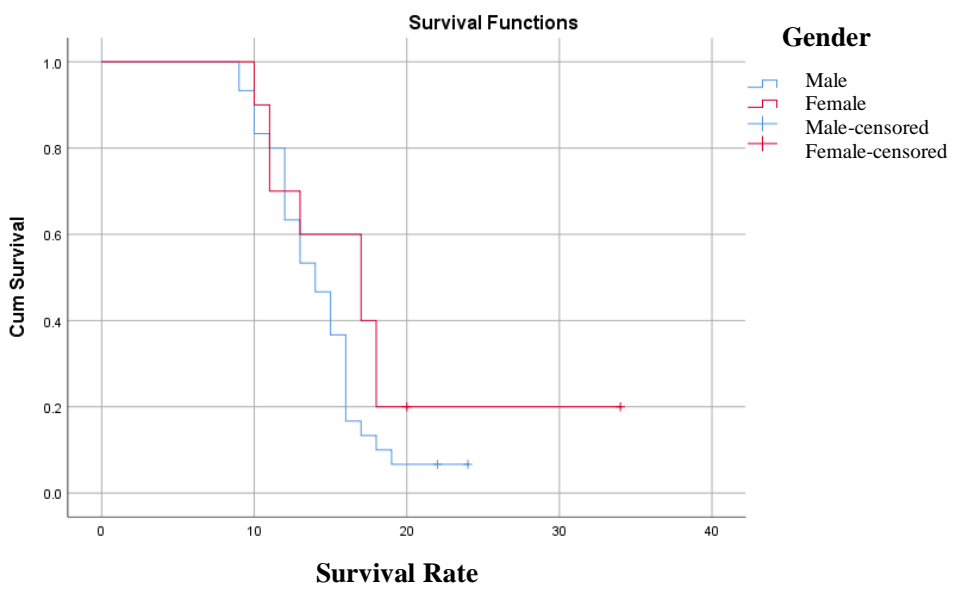

Figure 2. Kaplan-Meier Graph Showing 2-years Survival Rate by Gender

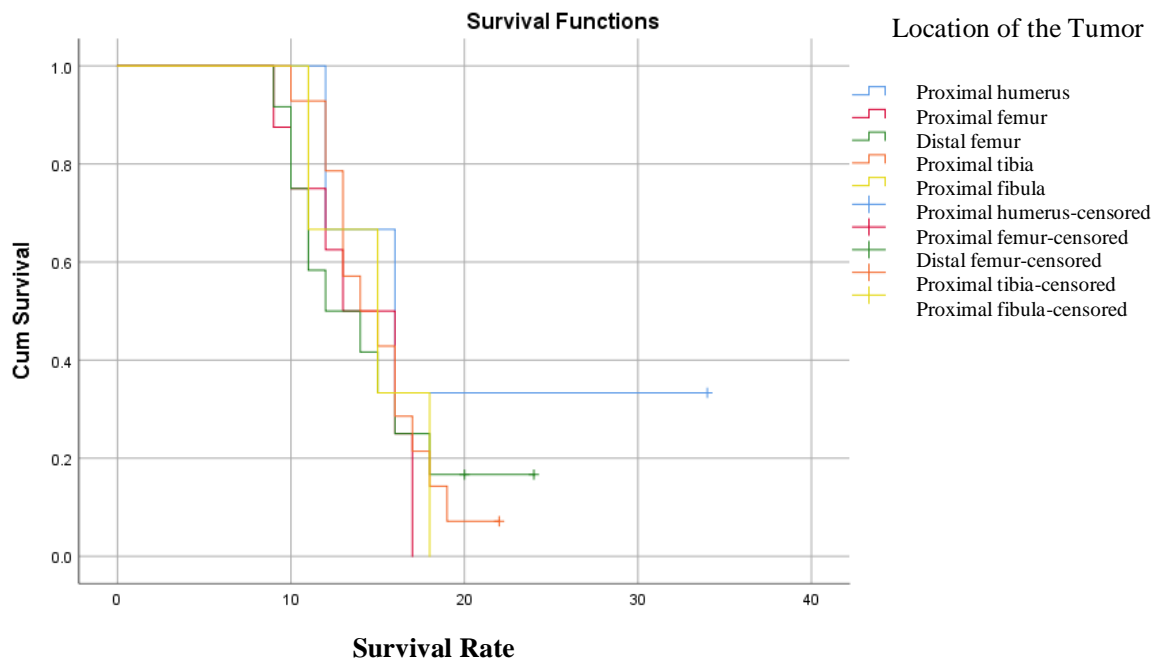

Figure 3. Kaplan-Meier Graph Showing 2-years Survival Rate by Location of the Tumor 


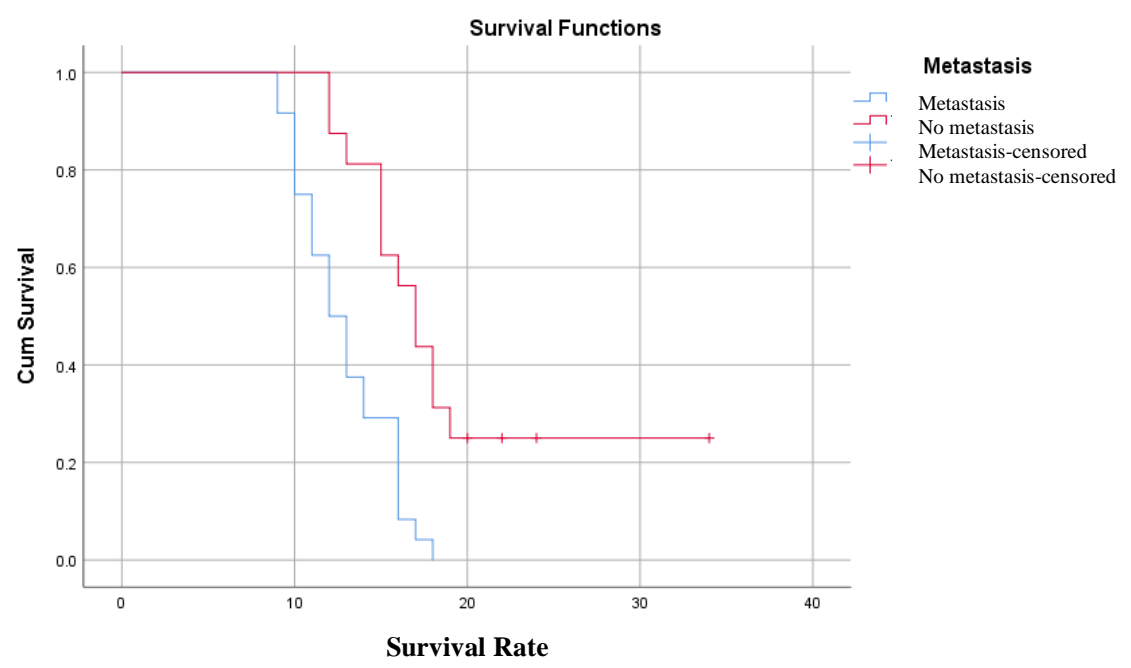

Figure 4. Kaplan-Meier Graph Showing 2-years Survival Rate by Metastasis / No metastasis

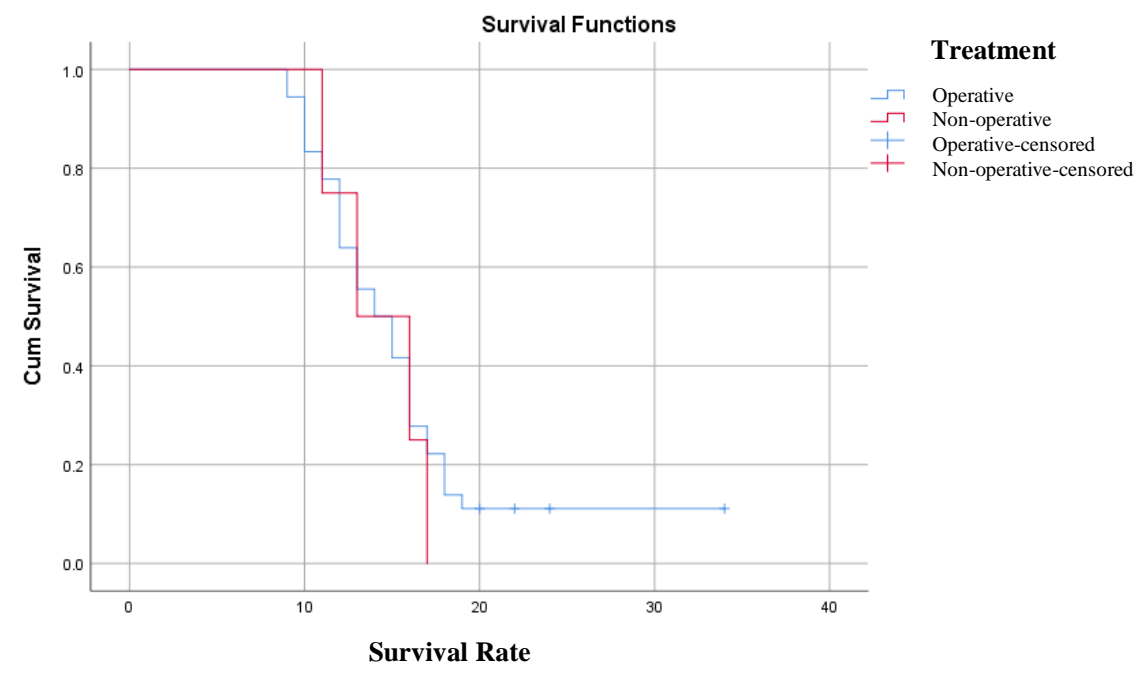

Figure 5. Kaplan-Meier Graph Showing 2-years Survival Rate by Treatment

In relation to the NLR value, 1 sample (2.5\%) with low NLR value showed a survival rate of more than 2 year, while 95 samples (97.5\%) had less than 2-year survival rate. The mean survival rate for samples with significantly lover NLR value has a survival rate of \pm 29 months, while samples with lower NLR value had a mean survival rate of \pm 13 months. 


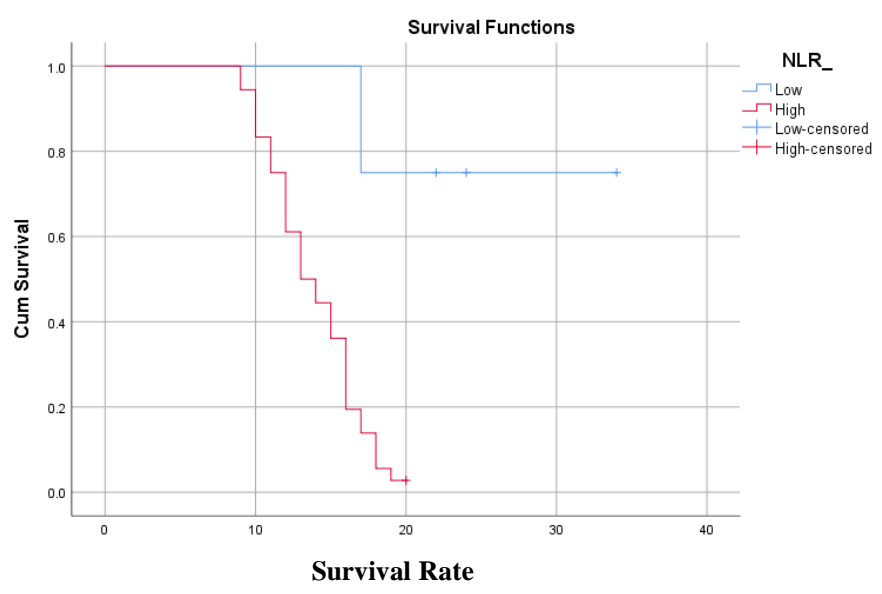

Figure 6. Kaplan-Meier Graph Showing 2-years Survival Rate by NLR value

In relation to the PLR value, 1 sample $(2.5 \%)$ with a low PLR value showed a survival of more than 2 years, while 39 samples $(97.5 \%)$ showed a PLR value of less than 2 years. The mean survival rate of samples with low PLR value is \pm 17 months, longer than samples with higher PLR value $( \pm 14$ months). Results are show in figure 8.

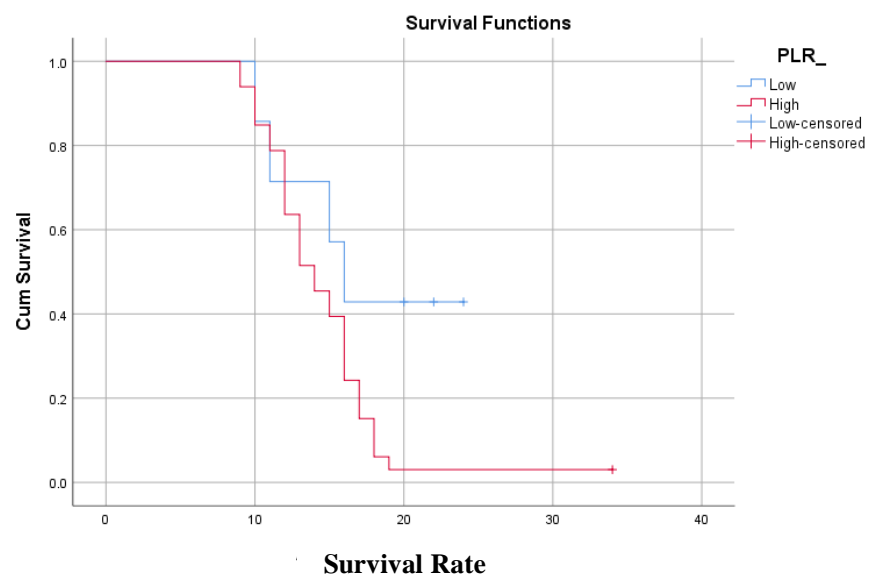

Figure 7. Kaplan-Meier Graph Showing 2-years Survival Rate by PLR value

From the results of ROC analysis obtained 2 variables have a deviation of 45 degrees, which means that it has minimum predictive capability in predicting Osteosarcoma prognosis. The variables consist of NLR (AUC: 0.972 / p-value: 0.002) and PLR (AUC: 0.944 / p-value 0.004) (Figure 8 and Table 2). From these observable variables, NLR and PLR are the best predictor abilities in predicting Osteosarcoma prognosis (AUC> 0.90 means is has incredibly good predictive ability). 


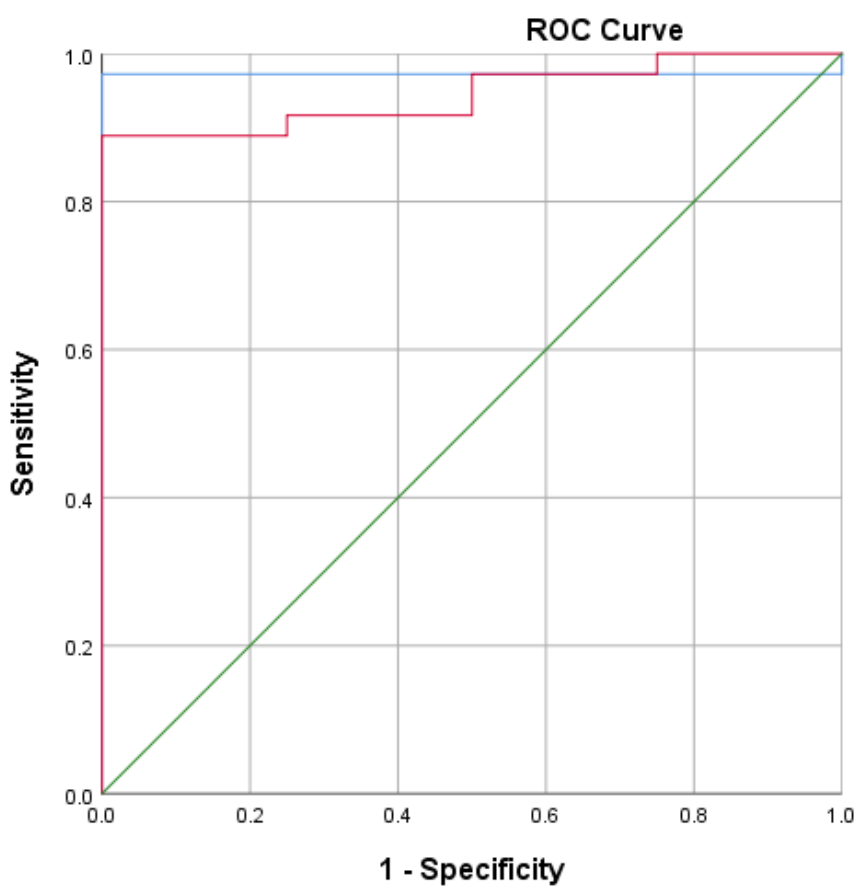

Source of the

Curve

NLR

- PLR

- Reference Line

Figure 8. ROC Curve Predictors for Osteosarcoma

Table 2 shows that using ROC analysis, two variables (NLR and PLR) show a deviation of $45^{\circ}$ which means that both variables are good predictor for osteosarcoma prognosis prediction $(0,944$, p-value 0.004 , CI 95\%, 0.871-1.000).

Table 2. Areas Under the Curve (AUC) Parameter Predictors of Osteosarcoma

Asymptotic 95\% Confidence Interval

\begin{tabular}{llllll} 
Variable & Area & Std. Error & p-value & Lower Bound & Upper Bound \\
\hline NLR & 0.972 & 0.027 & 0.002 & 0.919 & 1.000 \\
PLR & 0.944 & 0.038 & 0.004 & 0.871 & 1.000 \\
\hline
\end{tabular}

a. Under the nonparametric assumption

b. Null hypothesis: true area $=0.5$

To analyze the sensitivity, specificity, PPV and NPV of NLR, in this study we used $2 \times 2$ table below.

\begin{tabular}{l|c|c|}
\multicolumn{1}{c}{+} & - \\
\hline 3 & 1 \\
\hline 1 & 35 \\
\hline
\end{tabular}

NLR has a sensitivity of $75 \%$, specificity of $07 \%$, positive predictive value of $75 \%$, and negative predictive value of $97 \%$. 
To analyze the sensitivity, specificity, PPV and NPV of NLR, in this study we used $2 \times 2$ table below.

\begin{tabular}{l|l|l|}
\multicolumn{1}{c}{+} & + & - \\
\cline { 2 - 3 }+ & 3 & 4 \\
\hline 1 & 32 \\
\hline
\end{tabular}

PLR has a sensitivity of $75 \%$, specificity of $89 \%$, positive predictive value of $43 \%$, and a negative predictive value of $97 \%$.

\section{Discussion}

Osteosarcoma is an osteoblastic malignant tumor, derived from primitive mesenchymal cells, which can differentiate towards osteoblasts, chondroblasts, and fibroblasts. Therefore, the microscopically osteoblastic, chondroblastic, and fibroblastic in addition to malignant osteoid are seen, which is the diagnostic basis of osteosarcoma.

In this study, a total of 40 patients was included as the sample. The youngest age is 7 years-old and the oldest is 73 years-old, and the mean age was 18 years-old. With a 2-year survival rate, the life expectancy of osteosarcoma patients aged 0-10 years is greater than other age groups.

The sex ratio varied between sets but was dominant in males (1.3: 1 to $1.6: 1)$. The incidence rate is higher in males due to a longer period of bone growth. In this study, 30 patients (75\%) were male, and 10 $(25 \%)$ were female. Based on sex, the mean survival of female osteosarcoma patients ( \pm 18 months) was longer than that of male osteosarcoma patients ( \pm 14 months).

In this study, the most common tumor locations were the proximal tibia, which accounts for 14 people (35\%), then the distal femur in 12 people (30\%), the proximal femur in 8 patients $(20 \%)$, the proximal humerus (7.5\%) and the proximal fibula (7.5\%) with each region consisting of 3 patients. The mean survival of osteosarcoma patients from the tumor site in the proximal humerus ( \pm 20 months) was longer than that of other tumor sites; the proximal femur ( \pm 14 months) and distal femur, proximal tibia, and proximal fibula which were relatively the same ( \pm 15 months). The 2-year survival rate of osteosarcoma patients with tumor locations on the humerus is greater than patients with another tumor locations.

Based on tumor spread, from 40 patients, there were 26 patients whose cancer has metastases and 14 patients without metastases. Of the 14 patients who had no metastases, only 1 person had a life expectancy of more than 2 years. The mean survival of osteosarcoma patients with metastases ( \pm 12 months) was lower than that of osteosarcoma patients without metastases ( \pm 20 months).

Out of the 40 patients studied, 1 person (2.5\%) received palliative care, 1 person $(2.5 \%)$ underwent core biopsy, 2 people (5\%) underwent open biopsy, 1 person (2.5\%) was performed FNAB, 24 people (60\%) received AKA, four-quarter amputation was done 1 person (2.5\%), performed limb salvage procedure was performed on 2 patients (5\%) and hip disarticulation in 8 patients (20\%). Of the 36 people (90\%) who underwent surgery, only 1 patient had a life expectancy of more than 2 years. The mean survival of osteosarcoma patients who were operated on ( \pm 16 months) was longer than that of osteosarcoma patients who were not operated on ( \pm 14 months).

ROC analysis was performed to see the incidence of osteosarcoma by observing AUC determine its significance. The AUC NLR value for osteosarcoma (0.972, p-value 0.002, 95\% CI, 0.919-1000) was 
obtained, and the NLR cut-off value was 1.41 (sensitivity $75 \%$; specificity $97 \%$ ). The results were like the research conducted by Liu (2016), which found the sensitivity and specificity value of NLR for osteosarcoma (0.663, p-value $0.000,95 \%$ CI $0.578-0.748)$ with an NLR cut-off value of 2.57 (50\% sensitivity, $82.6 \%$ specificity. Following the study above, in this study, the 2-year survival rate of osteosarcoma patients was higher, namely 29 months in patients with low NLR vs 13 months in osteosarcoma patients with high NLR values.

Thus, the ROC analysis was carried out on the PLR value to determine significance. The AUC PLR value for osteosarcoma (0.944, p-value 0.004, 95\% CI 0.871 - 1,000) and cutoff value of 97.477 (sensitivity $75 \%$, specificity 89\%) were obtained. Research by Xia (2016) obtained AUC (0.606, p-value 0.001 ) and a cutoff value of 122 (sensitivity 44.6\%, specificity 66.4\%). In a study by Liu (2016), it was found that the survival rate of osteosarcoma patients was 86 months with low PLR values vs 36 months in patients with high PLR values. The same thing was found in this study, where the 2-year survival rate of osteosarcoma patients was 17 months with low PLR values vs 14 months in patients with high PLR values. 


\section{References}

Ando K, Heymann MF, Stresing V, Mori K, Redini F, Heymann D. (2013). Current therapeutic strategies and novel approaches in osteosarcoma. Cancers (Basel). 5(2):591-616.

Bacci G, Ferrari S, Lari S. (2002). Osteosarcoma of the limb. Amputation or limb salvage in patients treated by neoadjuvant chemotherapy. J Bone Joint Surg Br; 84(1):88-92.

Cortes EP, Holland JF, Wang JJ. (2005). Amputation and adriamycin in primary osteosarcoma. Clin Orthop Relat R. (438):5-8.

Ebeid W, Amin S, Abdelmegid A. (2005). Limb salvage management of pathologic fractures of primary malignant bone tumors. Cancer Control;12(1):57- 61.

Lewis VO. (2007). What's new in musculoskeletaloncology. J Bone Joint Surg Am;89a(6):1399-407.

Mankin, H. J., Hornicek, F. J., Rosenberg, A. E., Harmon, D. C., \& Gebhardt, M. C. (2004). Survival Data for 648 Patients with Osteosarcoma Treated at One Institution. Clinical Orthopaedics and Related Research. 429, 286-291.

Messerschmitt PJ, Garcia RM, Abdul-Karim FW, Greenfield EM, Getty PJ. (2009). Genetics of Osteosarcoma. J Am Acad Orthop Sur. 17(8):515-27.

Raymond A, Ayala A, Knuutila S. (2002). Conventional osteosarcoma. In: Fletcher CDM, Unni K, Mertens F, eds. World Health Organization Classification of Tumors

Ritter J, Bielack SS. (2010). Osteosarcoma. Ann Oncol;21:320-5.

Salter. (1999). Neoplasm of musculoskeletal tissues. Textbook of disorder and injuries of musculoskeletal system. Philadelphia: Lippincott-Williams-Wilkins.

Xia Wen K,Shen D, Lin Qing F. (2016). Prognostic Performance Of Pre-Treatment NLR and PLR in Patients Suffering From Osteosarcoma. World Journal of Surgical Oncology. Apr 29;14:127p. 